\title{
Rapid testing versus karyotyping in Down's syndrome screening: cost-effectiveness and detection of clinically significant chromosome abnormalities
}

\author{
Jean Gekas ${ }^{\star, 1,2}$, David-Gradus van den Berg ${ }^{3}$, Audrey Durand ${ }^{4}$, Maud Vallée ${ }^{2}$, Hajo Izaäk Johannes Wildschut ${ }^{3}$, \\ Emmanuel Bujold ${ }^{5}$, Jean-Claude Forest ${ }^{6}$, François Rousseau ${ }^{7}$ and Daniel Reinharz ${ }^{4}$
}

In all, $\mathbf{8 0} \%$ of antenatal karyotypes are generated by Down's syndrome screening programmes (DSSP). After a positive screening, women are offered prenatal foetus karyotyping, the gold standard. Reliable molecular methods for rapid aneuploidy diagnosis (RAD: fluorescence in situ hybridization (FISH) and quantitative fluorescence PCR (QF-PCR)) can detect common aneuploidies, and are faster and less expensive than karyotyping. In the UK, RAD is recommended as a standalone approach in DSSP, whereas the US guidelines recommend that RAD be followed up by karyotyping. A cost-effectiveness (CE) analysis of RAD in various DSSP is lacking. There is a debate over the significance of chromosome abnormalities (CA) detected with karyotyping but not using RAD. Our objectives were to compare the CE of RAD versus karyotyping, to evaluate the clinically significant missed CA and to determine the impact of detecting the missed CA. We performed computer simulations to compare six screening options followed by FISH, PCR or karyotyping using a population of 110948 pregnancies. Among the safer screening strategies, the most cost-effective strategy was contingent screening with QF-PCR (CE ratio of \$24084 per Down's syndrome (DS) detected). Using karyotyping, the CE ratio increased to $\$ 27898$. QF-PCR missed only six clinically significant CA of which only one was expected to confer a high risk of an abnormal outcome. The incremental CE ratio (ICER) to find the CA missed by RAD was $\$ 66608$ per CA. These costs are much higher than those involved for detecting DS cases. As the DSSP are mainly designed for DS detection, it may be relevant to question the additional costs of karyotyping.

European Journal of Human Genetics (2011) 19, 3-9; doi:10.1038/ejhg.2010.138; published online 15 September 2010

Keywords: Down's syndrome; prenatal diagnosis; chromosome abnormalities

\section{INTRODUCTION}

About $80 \%$ of antenatal cytogenetic referrals are in the context of a screening program for DS. ${ }^{1}$ In the last 15 years, major advancements have been made in prenatal screening for DS and prenatal diagnostic testing (amniocentesis or chorionic villus sampling) is offered only to pregnant women at high risk, ${ }^{2,3}$ of whom about $7 \%$ reveal a chromosomal abnormality (CA). ${ }^{4}$

The screening methods that identify women at high risk of having a foetus with DS include biochemical or ultrasonography markers (or both) combined with age-related algorithms. ${ }^{1}$ Similar to worldwideadvised procedures, ${ }^{5,6}$ six screening options consistent with US and Canadian guidelines are available: ${ }^{7,8}$ quadruple, combined, integrated and serum-only integrated tests, as well as stepwise sequential (sequential) and contingent sequential (contingent) screening approaches.

After a positive prenatal screening test, women are usually offered foetal karyotyping, which is considered as the gold standard to confirm the presence or absence of CA by counting the number of chromosomes and looking for structural changes. ${ }^{1}$ However, the main limitation of karyotyping remains the requirement for cell culture, resulting in a delay of 10-14 days for test results in many clinical genetic laboratories. ${ }^{9}$ Reliable ${ }^{10-13}$ molecular methods that are faster $(\leq 1-3 \text { days })^{14}$ and less expensive than karyotyping ${ }^{15-17}$ have been developed to detect common aneuploidies, which account for $>80 \%$ of the clinically relevant $\mathrm{CA},{ }^{14}$ although they do not provide a full photographic display of all chromosome pairs. Such methods include interphase FISH and QF-PCR, collectively referred to as RAD. ${ }^{18}$

There is still no consensus on the most cost-effective strategy that should be implemented to diagnose affected foetuses in DS screening programs. For some authors, the implementation of RAD services for all prenatal samples is considered to be a major step towards the optimisation of prenatal services. ${ }^{17}$ For the UK government ${ }^{19}$ and the UK National Screening Committee, new screening programs for DS need not include karyotyping and can offer diagnosis with RAD as a standalone approach. ${ }^{1}$

Nevertheless, a joint statement by the American College of Medical Genetics and the American Society for Human Genetics ${ }^{20}$ reaffirmed that all RAD test results must be followed up with karyotyping. ${ }^{1}$ Reports $^{11,16,21,22}$ indicate that $15-30 \%$ of CA detected by karyotyping

${ }^{1}$ Centre de recherche du CHUQ, Service de Génétique Médicale, Unité de Diagnostic Prénatal, Département de pédiatrie, Université Laval, Québec City, Québec, Canada; ${ }^{2}$ Laboratoire de Cytogénétique, Centre hospitalier universitaire de Québec (CHUQ), Département de biologie mé dicale, Québec City, Québec, Canada; ${ }^{3}$ Department of Obstetrics and Gynaecology, Erasmus University Medical Center, Rotterdam, The Netherlands; ${ }^{4}$ Département de médecine sociale et préventive, Laboratoire de Simulations des Dépistages, Faculté de Médecine, Université Laval, Québec City, Québec, Canada; ${ }^{5}$ Département d'obstétrique-gynécologie, Faculté de Médecine, Université Laval, Québec City, Québec, Canada; ${ }^{6}$ Centre de recherche du CHUQ, Département de biologie médicale, Faculté de Médecine, Université Laval, Québec City, Québec, Canada; ${ }^{7}$ CanGèneTest Research consortium, Centre de recherche du CHUQ, Département de biologie médicale, Faculté de Médecine, Université Laval, Québec City, Québec, Canada

*Correspondence: Professor J Gekas, Centre Hospitalier de I'Université Laval (CHUL), 2705, boulevard Laurier, bureau RC-9300, Sainte-Foy, Québec city, Québec G1V 4G2, Canada. Tel: +1 4185254444 ext: 48114; Fax: +1 418 6542748; E-mail: jean.gekas@mail.chuq.qc.ca

Received 26 March 2010; revised 22 June 2010; accepted 9 July 2010; published online 15 September 2010 
would not be detected by $\mathrm{RAD},{ }^{1}$ but the number of these $\mathrm{CA}$ with a risk of adverse outcome above background levels are much lower, ${ }^{1}$ and the relevance of diagnosing them via DS screening programs is debated owing to their clinical significance ${ }^{1,16-18}$

Actually, there is insufficient data available to resolve this question. Grimshaw et al ${ }^{15}$ noted that most investigations of these molecular tests focused on test errors rather than on CE analysis. So far, published reports addressing the $\mathrm{CE}$ of RAD versus full karyotyping have not estimated their use in relation with the various screening options that would comply with current guidelines for DS, ${ }^{15,16}$ and concluded that RAD would be less cost-effective than karyotyping owing to the cost of clinically significant CA missed by RAD, although the screening programs are not designed to detect them.

Given the numerous screening options to compare, any single empirical or clinical study is unlikely to evaluate all available strategies. Computer simulations are an elegant alternative to identify which strategy is likely to be the most cost-effective. ${ }^{23,24}$ Using simulations, we recently reported the impact of various first-trimester risk cutoffs for three different screening strategies combining first- and secondtrimester analyses. ${ }^{25}$

In the current study, we performed simulations using data from the Serum, Urine and Ultrasound Screening Study (SURUSS) ${ }^{23,26}$ for DS prenatal screening and from Caine et $a l^{1}$ for the expected CA missed by $\mathrm{RAD}$ with a potential level of adverse outcome, to: (i) compare the $\mathrm{CE}$ of three approaches to diagnose CA (karyotyping, FISH or QF-PCR) combined with six DS screening options, matched to worldwide-advised procedures ${ }^{5-8}$ (quadruple, combined, integrated and serum integrated tests, sequential and contingent screening approaches) for the detection of DS through prenatal screening programs; (ii) estimate their performance in identifying clinically significant CA; (iii) determine the impact of detecting missed CA by $\mathrm{RAD}$ in DS screening programs by calculating the ICER if karyotyping is performed.

\section{METHODS}

\section{Design, data, screening options and end points}

Using the modelling approach previously developed ${ }^{25}$ we performed computer simulations to estimate the performance of six screening options recommended by guidelines in the US and Canada (quadruple, combined, integrated and serum integrated tests, sequential and contingent screening approaches). ${ }^{7,8} \mathrm{We}$ evaluated these six screening options combined with three diagnostic approaches (karyotyping, FISH or QF-PCR) for end points that cover the main outcomes in DS prenatal screening. ${ }^{23,24}$ This yielded 18 potential screening algorithms. Figure $1 \mathrm{a}$ and $\mathrm{b}$ presents a simplified version of the decision model. The end points include: (1) the global costs; (2) the false-positive rate, which defines the number of scheduled prenatal diagnosis tests (amniocentesis or chorionic villus sampling); (3) the number of procedure-related euploid miscarriages; (4) the CE ratios (costs per DS detected); (5) the missed CA by RAD and (6) the ICER to detect them by karyotyping.

The virtual population comprised 110948 pregnancies corresponding to the number of pregnancies in 2001 in the province of Quebec. ${ }^{25}$ It allowed the generation of a virtual population with characteristics related to the prevalence and clinical course of non-DS or DS pregnancies, and a maternal age distribution identical to that found in the population of Quebec, and to simulate the costs and outcomes of all options under consideration.

To evaluate the impact of screening tests based on the gold standard of prenatal care, only amniocentesis was used for prenatal diagnostic testing following second-trimester screening results. However, for women who were tested positive in the first-trimester screening, a trans-abdominal chorionic villous sampling (CVS) karyotyping was considered.

The screening markers and procedures used are shown in Table 1. All input variables for simulations (screening procedures and expected CA) and their sources are presented in Tables 2, 3 and 4.
The number of DS pregnancies shown in Table 3 represents all DS cases in pregnancies registered for the population at each pregnancy period in 2001. The rates of DS pregnancy losses seemed to be more important than in other series $\left(43 \%{ }^{27}\right.$ and $30 \%{ }^{28}$ ), because part of these DS pregnancies are comprised in the voluntary pregnancy terminations in the first and second trimesters observed in our population in $2001 .^{25}$

\section{CA missed by RAD}

Because the clinical significance (ie, preventable morbidity and mortality) of detecting CA in perinatal and live born babies depends on the type of CA, we used results from Caine $e t a l^{1}$ to simulate the number and type of expected $\mathrm{CA}$ in our population, to evaluate $\mathrm{CA}$ missed by $\mathrm{RAD}$ and to estimate their potential level of adverse outcome. The study by Caine et al constitutes the largest published retrospective cytogenetic audit, assessing 142605 prenatal diagnoses. In the simulations, the CA were classified as detectable, sometimes detectable or undetectable by RAD and for clinical significance, as background risk, low-to-high risk and high risk of adverse outcome (Table 4). Also, we estimated the additional costs of detecting clinically significant CA missed by RAD by calculating the $\mathrm{ICER}^{24}$ obtained if full karyotyping is performed.

\section{Test performance}

The distribution of SURUSS marker results in DS-affected and DS-unaffected pregnancies was used to determine the screening test performances. ${ }^{23,26}$ All false-positive rates and risk cutoffs were standardised to the same gestational age (11-week gestation) for first-trimester measurements. To compare firsttrimester versus second-trimester screening procedures on a common basis, we used a fixed detection rate, which is more applicable than a fixed false-positive rate because applying the latter to the first-trimester as compared with the second-trimester results in a different detection rate, notably from the spontaneous losses of DS foetuses between the first and second trimesters. ${ }^{29}$ We chose the $90 \%$ detection rate as it is that used in the literature for test performances and cutoffs specified for all the screening tests (integrated, ${ }^{26}$ sequential and contingent screening tests ${ }^{23}$ ) used in the first and second trimesters. Also, as of April 2010, the UK National Screening Committee targets a detection rate of more than $90 \%{ }^{6}$

\section{Costs}

In Canada, in accordance with the Canadian Health Care Act, all medical necessary services are provided under the public healthcare system and are free of charge. Costs from provincial technical units were used for laboratory and imaging tests as previously detailed. ${ }^{25}$ Costs reported in Table 2 for screening tests do not reflect the cost of any single procedure but the mean cost for all medical necessary services provided for each screening option. Items considered for costing included screening costs as well as healthcare and medical services related to the following outcomes: birth, spontaneous miscarriage, elective abortion or procedure-related euploid miscarriages. Costs are expressed in Canadian dollars (CAD). The average exchange rate in 2007 was: 1.0748 $\mathrm{CAD}=1.00$ USD $=0.73$ EUR.

\section{$\mathrm{CE}$ analysis and confidence intervals}

All measured costs occurred within 1 year; therefore, there was no need to discount costs and effects over time. ${ }^{24}$ Univariate sensitivity analyses ${ }^{25}$ were performed on the rate of consent to participate in prenatal screening (65 and $80 \%)$, the rates of foetal loss from CVS $(0.5,1$ and $2 \%)$ and the rates of foetal loss from amniocentesis ( 1 and 1.5\%). Moreover, the sensitivities and falsepositive rates of DS screening strategies varied over the ranges achieved in the SURUSS trial. ${ }^{23,26}$ To generate $95 \%$ confidence intervals (CI 95\%) for global cost estimates, a bootstrap method was used as previously described ${ }^{25}$.

\section{RESULTS}

The global cost analysis results, including the outcomes as a function of the screening strategy and diagnostic tool that were used, are summarised in Table 5. The CE ratio to detect DS cases (with the same screening strategy and QF-PCR for diagnosis), the number of CA 
a

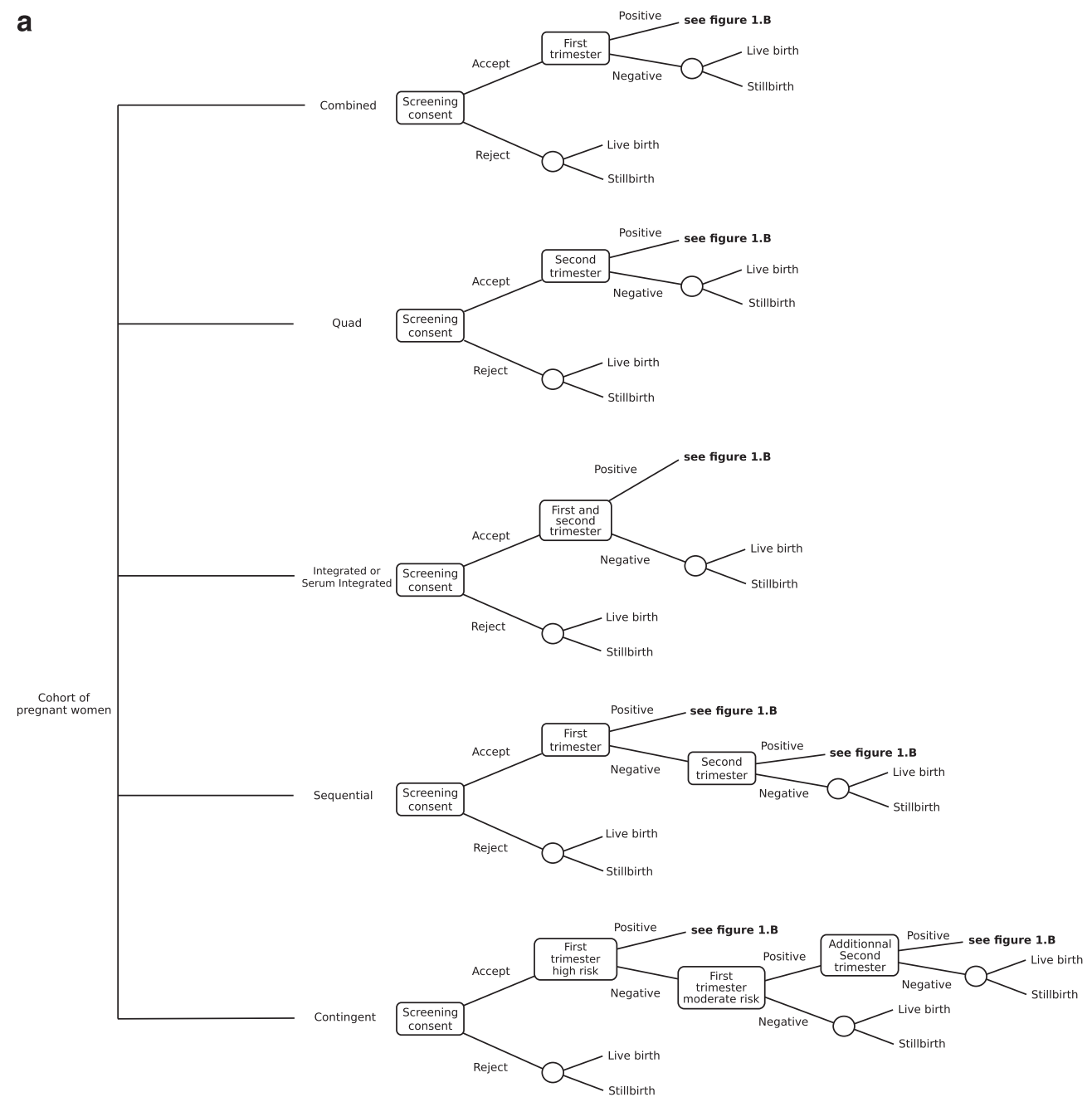

b

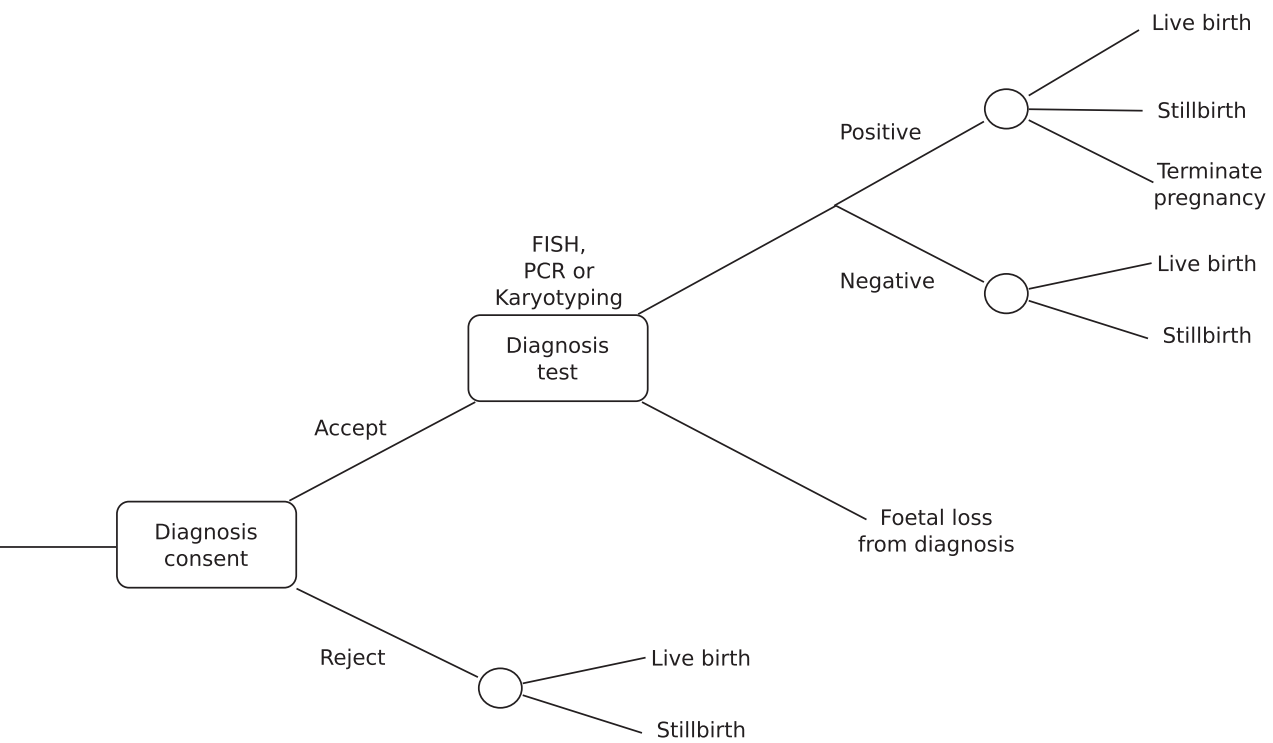

Figure 1 Simplified version of the decision trees: (a) algorithms for screening options; (b) algorithms for the diagnosis procedure. Not shown in this simplified depiction, but included in our model, is the possibility that miscarriage occurs before testing or after releasing results.

missed by RAD according to the risk category of an abnormal outcome and the ICER for detecting them by karyotyping are reported in Table 6.
Costs and main outcomes of DS screening options

With the same DS detection rate, all screening strategies cost less when using QF-PCR. Using the FISH test was associated with higher 
Table 1 Definitions of screening procedures

Combined test

First-trimester test based on combining nuchal translucency measurement (NT, an ultrasound measurement of the width of an area of translucency at the back of the foetal neck early in pregnancy) with free human chorionic gonadotropin (free $\beta$-hCG), pregnancy-associated plasma protein A (PAPP-A) and maternal age.

Quadruple test $\quad$ Second-trimester test based on the measurement of AFP, uE3, free $\beta$-hCG (or total hCG) and inhibin-A together with maternal age.

Integrated test The integration of measurements performed at different times of pregnancy into a single test result. Unless otherwise qualified, 'integrated test' refers to the integration of NT and PAPP-A in the first trimester with the quadruple test markers in the second. The first-trimester screening marker results are not analyzed until the second-trimester markers are evaluated, at which point they are both assessed together.

Serum integrated test ${ }^{42}$ A variant of the integrated test without NT (using PAPP-A in the first trimester and quadruple test markers in the second trimester).

Sequential screening ${ }^{23}$ Screening in which a first-trimester test is performed (NT, free $\beta$-hCG and PAPP-A) and the result is interpreted immediately. If this is positive, a diagnostic test is offered (chorionic villous sampling, CVS), but if it is not positive, second-trimester serum markers are measured (quadruple test markers) and the first-trimester markers are reused to form an integrated test.

Contingent screening ${ }^{23}$ Screening in which a first-trimester test (NT, free $\beta$-hCG and PAPP-A) is used to triage the population of women screened into three groups: one group (high-risk screen-positive) that is immediately offered a diagnostic test (CVS), a second group (screen-negative) that receives no further screening and a third intermediate (or low-risk screen-positive) group that has second-trimester markers measured (quadruple test markers) and the first-trimester measurements reused to form an integrated test.

Risk cutoff The risk or likelihood of the condition being present in the foetus above which a prenatal diagnosis test is proposed.

Table 2 Analysis input variables

\begin{tabular}{lcl}
\hline & Cost (\$) & References \\
\hline Input costs of screening or diagnostic tests or events & & \\
Integrated test & 65 & 25 \\
Sequential screening & 105 & 25 \\
Contingent screening & 55 & 25 \\
Serum integrated test & 35 & 25 \\
Quadruple test & 25 & 25 \\
Combined test & 40 & 25 \\
Consulting with a genetic counselor & 73.90 & 25 \\
CVS + diagnostic procedure karyotyping & 876 & 25 \\
Amniocentesis + diagnostic procedure karyotyping & 500 & 25 \\
CVS + diagnostic procedure QF-PCR (Aneufast) & 198 & 25,43 \\
Amniocentesis + diagnostic procedure & 198 & 25,43 \\
QF-PCR (Aneufast) & & \\
CVS + diagnostic procedure FISH & 422 & 25,43 \\
Amniocentesis + diagnostic procedure FISH & 422 & 25,43 \\
Termination of pregnancy & 1357.33 & 25 \\
& & \\
Input variables of screening tests with & False-positive & References \\
a 90\% detection rate & rate (\%) & \\
\hline Integrated test (1/230 cutoff) & 2.11 & $30,35,44$ \\
Sequential screening (1/9 cutoff, first trimester) & \\
Contingent screening (1/9 cutoff, first trimester) & 2.25 & 23,25 \\
Serum integrated test (1/355 cutoff) & 2.42 & 23,25 \\
Quadruple test (1/545 cutoff) & 5.30 & 26,30 \\
Combined test (1/625 cutoff) & 10.60 & 26,30 \\
& 8.40 & 30,35 \\
Different diagnostic tools & 99.40 & 45 \\
\hline Karyotyping & 99.30 & 14 \\
QF-PCR & 98.70 & 14 \\
FISH & & \\
\hline & Sensitivity (\%) & References \\
\hline
\end{tabular}

Abbreviations: \$, Canadian dollars; CVS, chorionic villous sampling.

aSequential and contingent screening tests consist of a sequence of analysis with many possible cutoff combinations, notably in the first trimester ${ }^{23}$. A risk cutoff is the risk or likelihood of the condition being present in the foetus. A woman was classified as positive if her risk estimate was equal to or greater than the corresponding specific cutoff level. Given the published data, for sequential and contingent screening tests, the first-trimester high-risk cutoff we applied was one in nine as previously advised ${ }^{25}$ and in the contingent screening approach, the lower risk cutoff used in the first test was 1 in $2000 .{ }^{23}$
Table 3 Demographic characteristics of the population and assumptions $^{\mathrm{a}}$

\begin{tabular}{l} 
Population simulated \\
\hline Total pregnant women \\
DS pregnancies at first trimester \\
DS pregnancies at second trimest \\
DS pregnancies at third trimester \\
DS babies at birth \\
\\
Pregnant women's age distribution \\
$<20$ \\
$20-24$ \\
$25-29$ \\
$30-34$ \\
$35-39$ \\
$40-44$ \\
$45 \geq$
\end{tabular}

Number References

$110948 \quad 25$

$290 \quad 25,27,28$

$190 \quad 25,27,28$

$140 \quad 25,27,28$

$13125,27,28$

Events before or after screening and diagnostic intervention

$9008 \quad 25$

$24987 \quad 25$

$33421 \quad 25$

$27320 \quad 25$

$13135 \quad 25$

$2925 \quad 25$

$152 \quad 25$

\begin{tabular}{lll}
\hline Consent to participate in prenatal screening & 70 & 25 \\
Consent for amniocentesis or CVS with & 90 & 25 \\
screening positive & & \\
Foetal loss from amniocentesis & 0.5 & $25,37,46-49$ \\
Foetal loss from CVS & 1.6 & $25,37,46-49$ \\
Proportion who terminated pregnancy with foetal DS & 90 & 25 \\
\hline
\end{tabular}

Abbreviations: DS, Down's syndrome; CVS, chorionic villous sampling.

aData simulations were performed on a virtual population of 110948 pregnancies with demographic (maternal age distribution), genetic and phenotypic (regarding DS) characteristics of the Quebec population in the year 2001.25

costs than using QF-PCR (Table 5). When considering only the safer screening strategies (ie, with a number of procedure-related euploid miscarriages induced $<10$ per 100000 pregnancies), the most cost-effective strategy was the contingent screening associated with QF-PCR (global cost of \$2 497610 in Table 5) and CE ratio of \$24 084 per DS detected (Table 6). The CE ratio of this screening strategy associated with full karyotyping would be $\$ 27898$ per DS detected. The global cost and CE ratio of the least cost-effective screening 
Table 4 Classification of expected chromosome abnormalities ${ }^{a}$

Type of chromosome abnormalities

Down's syndrome T21

Edwards' syndrome T18

Patau's syndrome T13

Triploidy

Tetraploidy

Balanced structural rearrangement (inherited)

Robertsonian translocation (de novo or of unknown origin)

Marker chromosome (inherited)

45,X; 47,XXX; 47,XXY; 47,XYY

$45, X$ mosaic; $47, X X Y$ mosaic or sex chromosome mosaic

$45, X$ structurally abnormal $X$ or structurally abnormal $X / Y$ chromosome

Balanced structural rearrangement (de novo or of unknown origin)

Marker chromosome (de novo or of unknown origin)

Other autosomal trisomy

Unbalanced structural rearrangement

Detectability by karyotyping ${ }^{\mathrm{b}} \quad$ Detectability by $R_{A} D^{\mathrm{b}} \quad$ Risk categories of clinical significance ${ }^{\mathrm{c}}$

Abbreviation: RAD, rapid aneuploidy diagnosis.

aClassification according to Caine $\mathrm{et}^{\mathrm{al}} \mathrm{l}$ depending on their type, their detectability by karyotyping or RAD, and their clinical significance.

betectability: 1 =detectable; $2=$ sometimes detectable; 3 =undetectable.

'Risk categories of clinical significance: 1 =background; 2 =low-to-high risk; $3=$ high risk.

Table 5 Global costs, amount of diagnostic procedures induced and number of procedure-related euploid miscarriages

\begin{tabular}{|c|c|c|c|c|c|c|c|}
\hline \multirow[b]{2}{*}{ Screening strategies } & \multirow[b]{2}{*}{$Q F-P C R$} & \multirow{2}{*}{$\begin{array}{c}\text { Global costs }^{a} \\
\text { FISH }\end{array}$} & \multirow[b]{2}{*}{ Karyotyping } & \multirow{2}{*}{$\begin{array}{c}\text { Difference in karyotyping } \\
\text { and } Q F-P C R^{\mathrm{b}}\end{array}$} & \multicolumn{2}{|c|}{ Number of diagnostic procedures } & \multirow{2}{*}{$\begin{array}{l}\text { Procedure-related } \\
\text { euploid miscarriages }\end{array}$} \\
\hline & & & & & Amniocentesis & CVS & \\
\hline Integrated & $3.06_{(0.0016)}$ & $3.28_{(0.0016)}$ & $3.36_{(0.0020)}$ & 0.294 & 908(903-913) & & $4_{(3.4-4.3)}$ \\
\hline Sequential & $3.38_{(0.0028)}$ & $3.62_{(0.0033)}$ & $3.74_{(0.0048)}$ & 0.358 & $883_{(878-889)}$ & $102_{(100-103)}$ & $5_{(4.4-5.3)}$ \\
\hline Contingent & $2.50_{(0.0021)}$ & $2.76_{(0.0029)}$ & $2.90_{(0.0035)}$ & 0.399 & $995(989-1001)$ & $111_{(109-112)}$ & $6_{(5.3-6.9)}$ \\
\hline Serum integrated & $2.14_{(0.0024)}$ & $2.69_{(0.0038)}$ & $2.88_{(0.0043)}$ & 0.739 & $2.293(2.285-2.300)$ & & $15_{(14-16)}$ \\
\hline Quadruple & $2.08_{(0.0033)}$ & $3.18_{(0.0057)}$ & $3.55_{(0.0072)}$ & 1.464 & $4.537_{(4.524-4.550)}$ & & $22(21-23)$ \\
\hline Combined $^{8.4 \%}$ & $2.78_{(0.0032)}$ & $3.62_{(0.0053)}$ & $5.35(0.0112)$ & 2.568 & & $3.760_{(3.746-3.772)}$ & $70_{(68-72)}$ \\
\hline
\end{tabular}

Confidence intervals are given in brackets.

${ }^{\mathrm{a} G l o b a l}$ costs are expressed in MCAD (million in Canadian dollars).

bifference of costs between karyotyping and QF-PCR are expressed in MCAD.

Table 6 CE ratios to detect DS, number of chromosome abnormalities missed by RAD, and their ICER

\begin{tabular}{|c|c|c|c|c|}
\hline \multirow[b]{2}{*}{ Screening strategies } & \multirow[b]{2}{*}{$C E$ ratios to detect $D S$ by $Q F-P C R$} & \multicolumn{2}{|c|}{ Number of missed $C A$ by $R A D$} & \multirow{2}{*}{$\begin{array}{c}\text { ICER to detect CA of groups } \\
2 \text { and } 3 \text { by karyotyping }\end{array}$} \\
\hline & & Low-to-high (2) and high risk (3) & High risk (3) & \\
\hline Integrated & 34293 & 5 & 1 & 59377 \\
\hline Sequential & 33227 & 5 & 1 & 71646 \\
\hline Contingent & 24084 & 6 & 1 & 66608 \\
\hline Serum integrated & 24103 & 13 & 2 & 59034 \\
\hline Quadruple & 23754 & 25 & 5 & 59077 \\
\hline Combined $^{8.4 \%}$ & 24853 & 20 & 4 & 125278 \\
\hline
\end{tabular}

Abbreviations: CE, cost-effectiveness; DS, Down's syndrome; CA, chromosome abnormality; RAD, rapid aneuploidy diagnosis; ICER, incremental cost-effectiveness ratio.

strategy (combined test using karyotyping) would be $\$ 5347554$ (Table 5) and $\$ 47358$ per DS detected, respectively.

The difference in global cost between FISH and QF-PCR varied from $\$ 844915$ for the combined test to $\$ 220404$ for the integrated screening (Table 5). The largest difference in global cost for 100000 pregnancies between QF-PCR and karyotyping was observed with the combined test, \$2567981 (\$5347554 with karyotyping and $\$ 2779573$ with QF-PCR). The highest and lowest numbers of procedure-related euploid miscarriages occurred with the combined test $(n=70)$ and the integrated test $(n=4)$, respectively (Table 5$)$.

\section{Missed CA}

Clinically significant CA were missed if the diagnosis was performed by RAD instead of karyotyping. However, when applied to 100000 pregnancies, the contingent screening method associated with QF-PCR missed only six clinically significant CA (belonging to risk categories 2 or 3 , ie, low-to-high and high risk), of which only one was expected to confer a high risk of an abnormal outcome (Table 6). The additional cost of finding these CA (ICER) by karyotyping instead of QF-PCR was much higher than the CE ratios to find DS cases regardless of the screening strategy considered (Table 6). Depending 
on which screening option is used, the ICER to find the CA missed by RAD ranged from $\$ 59034$ to $\$ 125278$ per CA (Table 6). Although FISH missed the same number of clinically significant CA as QF-PCR, it was associated with higher costs (Table 5).

\section{DISCUSSION}

\section{Limitations}

Although our study is based on computer modelling rather than prospective data, our results are strengthened by the use of empirical data and true healthcare costs. It is rather unlikely that a vast prospective clinical study comparing the 18 screening strategies studied could realistically be performed. There is strong evidence that computer simulations are a powerful alternative to such expensive and hard-to-manage large-scale clinical studies. ${ }^{25,30}$

All the reported CE ratios were computed in the context of the Quebec health care system and costs. However, with respect to cost comparisons, relative costs (ranking of different scenarios) usually lend themselves better to comparison. Also, given the robustness of our findings in the sensitivity analyses, the relative performance (or ranking) of various scenarios would likely be similar in other jurisdictions. Furthermore, the demographics of the simulated population are comparable to those found in other Western countries. Mean maternal age and the proportion of women over 35 years are comparable to the SURUSS $^{26}$ and the First- and Second-Trimester Evaluation of Risk trial (FASTER trial) ${ }^{31}$ populations, which are representative of women in the UK and the USA, respectively.

\section{RAD are the most cost-effective diagnostic tools}

Our results demonstrate that RAD are the most cost-effective diagnostic tools and, although FISH misses the same number of clinically significant CA as QF-PCR, it is associated with higher costs. As DS screening programs are mainly designed for DS detection, it may be relevant to question the additional costs of full karyotyping to detect other anomalies. Quality of life and anxiety measurements show a significantly increased health status after diagnosis with RAD, ${ }^{15}$ and $\mathrm{RAD}$ allows earlier decision making in cases where the foetus has a detected CA. ${ }^{32}$ Also, the anxiety generated after being diagnosed with a not requested and not clinically significant CA by karyotyping can be prevented by using RAD. ${ }^{33}$

Furthermore, it could be more cost-effective to promote the use of QF-PCR instead of FISH, which is generally the first choice in genetic laboratories, and to favour contingent screening, which this study suggests as being the most cost-effective screening strategy for DS and associated with a more acceptable rate of procedure-related euploid miscarriages.

The combined test was associated with the highest number of procedure-related euploid miscarriages, which are the main adverse outcomes that should be reduced in DS prenatal screening. ${ }^{34}$ This is certainly owing to the fact that CVS is associated with the highest rate of procedure-related euploid miscarriages in women who had tested positive in the first trimester. Furthermore, a higher number of unnecessary terminations could contribute to the observed excess of procedure-related euploid miscarriages. Indeed, too early, a diagnosis may induce an excess in unnecessary terminations of the DS cases screened, ${ }^{23}$ as a spontaneous miscarriage may occur between the first and second trimesters. ${ }^{28}$ Some women who were screened as positive in the first trimester actually prefer to wait for an amniocentesis in the second trimester to avoid these disadvantages of CVS. The falsepositive rate used for the combined test in our study was $8.4 \%$ for a detection rate of $90 \%$, as revised by the SURUSS research group. ${ }^{35}$ This agrees with the false-positive rate reported by Malone et $a l^{31}$ in the FASTER trial for first-trimester screening, as at 11 weeks, the observed false-positive rate was $3.8 \%$ for a detection rate of $85 \%$, and $18 \%$ for a detection rate of $95 \%$. As other prospective trials evaluating first-trimester screening have reported a better efficiency for this screening strategy (eg, 5\% false-positive rate for a $90 \%$ detection rate $^{36}$ ), we simulated the $\mathrm{CE}$ and the number of procedure-related euploid miscarriages using the latter values and even with these assumptions, we confirmed the costs of the combined test: \$4034243 (4024213-4044274) and 34 (33-35 for CI) for global cost (if used with karyotyping) and for the number of procedurerelated euploid miscarriages, respectively.

\section{RAD detect the majority of clinically significant CA}

Our findings show that only a very small number of clinically significant $\mathrm{CA}$ are missed when $\mathrm{RAD}$ is used as a standalone approach and that savings can be substantial depending on the screening strategy used. For most of the screening scenarios evaluated, the incidence of procedure-related euploid miscarriages, which represent foetal losses of normal babies, was higher than the number of missed CA by RAD, especially when only high-risk CA were considered. The choice of the screening strategy, especially when based on the falsepositive rate involved, could therefore have a more damaging impact than the use of RAD as a standalone diagnostic approach in DS screening programs.

\section{ICERs to detect clinically significant CA missed by RAD are higher} than the CE ratios to detect DS cases

We have shown that the additional costs to detect clinically significant CA missed by QF-PCR were much higher than the cost to detect cases of DS, ie, between 1.7-fold with the integrated test to 5-fold higher with the combined test. As the screening programs developed are only designed for DS screening or other common aneuploidies (trisomy 13 or 18) also detectable with $\mathrm{RAD}$, and as women are not always aware of the fact that other CA can be detected with karyotyping, ${ }^{1,33}$ taking on the additional costs involved for full karyotyping may be questionable.

Caine et $a l^{1}$ report that out of the 98166 amniotic fluids referred from DS screening programs, 293 substantial-risk CA would be missed if RAD had been used. Taking a procedure-related miscarriage rate of $0.5 \%,{ }^{25,37}$ these 98166 amniocentesis tests would have entailed 491 procedure-related miscarriages, ie, nearly twice the number of substantial risk CA missed by RAD. Also, it remains to be established whether withdrawal of karyotyping for pregnant women using prenatal screening programs for DS will have a substantial effect on the incidence of hitherto preventable morbidity and mortality in perinatal and live born babies, as previously stated by Caine et al. ${ }^{1}$ The incidence of birth defects are estimated at about 3.4\% in the general population $^{38}$ and, in $50 \%$ of cases, medical tests show normal results including karyotyping. ${ }^{39}$ Therefore, some recent studies support the use of novel diagnostic approaches for these patients with DNA arrays instead of conventional karyotyping. ${ }^{40}$ Thus, diagnostic confirmatory tools should perhaps focus on the original goal of DS prenatal screening programs: diagnosing DS cases.

Nevertheless, pregnant women who benefit from an amniocentesis also take the risk of a procedure-related miscarriage. Therefore, dimensions such as woman's values that are challenged by the additional information provided by karyotyping (including the information brought by a normal result), by the unexpected diagnosis of $\mathrm{CA}$, and the anxiety generated by the birth of a child with clinically significant CA that could have been detected by karyotyping should all be considered. ${ }^{41}$ 


\section{CONFLICT OF INTEREST}

The authors declare no conflict of interest.

\section{ACKNOWLEDGEMENTS}

This work was supported by the Canadian Institutes for Health Research (Institutes of Genetics and Institutes of Health Services Research), the Canadian Agency for Drugs and Technologies in Health, and the Canadian Heart and Stroke Foundation through a grant to the CanGéneTest research consortium on genetic laboratory services.

1 Caine A, Maltby AE, Parkin CA, Waters JJ, Crolla JA: Prenatal detection of Down's syndrome by rapid aneuploidy testing for chromosomes 13,18 , and 21 by FISH or PCR without a full karyotype: a cytogenetic risk assessment. Lancet 2005; 366: 123-128.

2 Wald NJ, Hackshaw AK, Watt H: Nuchal translucency and trisomy 18. Prenat Diagn 1999; 19: 995-996.

3 Wapner R, Thom E, Simpson JL et al: First-trimester screening for trisomies 21 and 18. N Engl J Med 2003; 349: 1405-1413.

4 NEQAS: National External Quality Assessment Scheme in clinical cytogenetics. Annu Rep 2000

5 Boyd PA, Devigan C, Khoshnood B, Loane M, Garne E, Dolk H: Survey of prenatal screening policies in Europe for structural malformations and chromosome anomalies, and their impact on detection and termination rates for neural tube defects and Down's syndrome. BJOG 2008; 115: 689-696.

6 NSC: Fetal Anomaly Screening Programme - Screening for Down's Syndrome: UK NSC Policy recommendations 2007-2010: Model of Best Practice. UK: Department of Health, 2008.

7 ACOG Committee on Practice Bulletins: ACOG Practice Bulletin No 77: screening for fetal chromosomal abnormalities. Obstet Gynecol 2007; 109: 217-227.

8 Summers AM, Langlois S, Wyatt P, Wilson RD: Prenatal screening for fetal aneuploidy. J Obstet Gynaecol Can 2007; 29: 146-179.

9 Waters JJ, Waters KS: Trends in cytogenetic prenatal diagnosis in the UK: results from UKNEQAS external audit, 1987-1998. Prenat Diagn 1999; 19: 1023-1026.

10 Cirigliano V, Voglino G, Canadas MP et al: Rapid prenatal diagnosis of common chromosome aneuploidies by QF-PCR. Assessment on 18,000 consecutive clinical samples. Mol Hum Reprod 2004; 10: 839-846.

11 Hulten MA, Dhanjal S, Pertl B: Rapid and simple prenatal diagnosis of common chromosome disorders: advantages and disadvantages of the molecular methods FISH and QF-PCR. Reproduction 2003; 126: 279-297.

12 Mann K, Donaghue C, Fox SP, Docherty Z, Ogilvie CM: Strategies for the rapid prenatal diagnosis of chromosome aneuploidy. Eur J Hum Genet 2004; 12: 907-915.

13 Verma L, Macdonald F, Leedham P, McConachie M, Dhanjal S, Hulten M: Rapid and simple prenatal DNA diagnosis of Down's syndrome. Lancet 1998; 352: 9-12.

14 Shaffer LG, Bui TH: Molecular cytogenetic and rapid aneuploidy detection methods in prenatal diagnosis. Am J Med Genet C Semin Med Genet 2007; 145C: 87-98.

15 Grimshaw GM, Szczepura A, Hulten M et al: Evaluation of molecular tests for prenatal diagnosis of chromosome abnormalities. Health Technol Assess 2003; 7: 1-146.

16 Evans MI, Henry GP, Miller WA et al: International, collaborative assessment of 146,000 prenatal karyotypes: expected limitations if only chromosome-specific probes and fluorescent in-situ hybridization are used. Hum Reprod 1999; 14: $1213-1216$.

17 Mann K, Fox SP, Abbs SJ et al: Development and implementation of a new rapid aneuploidy diagnostic service within the UK National Health Service and implications for the future of prenatal diagnosis. Lancet 2001; 358: 1057-1061.

18 Boormans EM, Birnie E, Bilardo CM, Oepkes D, Bonsel GJ, van Lith JM: Karyotyping or rapid aneuploidy detection in prenatal diagnosis? The different views of users and providers of prenatal care. BJOG 2009; 116: 1396-1399.

19 UK Department of Health: Our Inheritance, Our Future: Realising the Potential of Genetics in the NHS. London: Stationery Office, 2003.

20 Test and Technology Transfer Committee: Technical and clinical assessment of fluorescence in situ hybridization: an ACMG/ASHG position statement. I. Technical considerations, Genet Med 2000; 2: 356-361.

21 Thein AT, Abdel-Fattah SA, Kyle PM, Soothill PW: An assessment of the use of interphase FISH with chromosome specific probes as an alternative to cytogenetics in prenatal diagnosis. Prenat Diagn 2000; 20: 275-280.

22 Thilaganathan B, Sairam S, Ballard T, Peterson C, Meredith R: Effectiveness of prenatal chromosomal analysis using multicolor fluorescent in situ hybridisation. BJOG 2000; 107: 262-266.
23 Wald NJ, Rudnicka AR, Bestwick JP: Sequential and contingent prenatal screening for Down syndrome. Prenat Diagn 2006; 26: 769-777.

24 Caughey AB: Cost-effectiveness analysis of prenatal diagnosis: methodological issues and concerns. Gynecol Obstet Invest 2005; 60: 11-18.

25 Gekas J, Gagne G, Bujold E et al: Comparison of different strategies in prenatal screening for Down's syndrome: cost effectiveness analysis of computer simulation. BMJ 2009; 338: b138.

26 Wald NJ, Rodeck C, Hackshaw AK, Walters J, Chitty L, Mackinson AM: First and second trimester antenatal screening for Down's syndrome: the results of the Serum, Urine and Ultrasound Screening Study (SURUSS). J Med Screen 2003; 10: 56-104.

27 Morris JK, Wald NJ, Watt HC: Fetal loss in Down syndrome pregnancies. Prenat Diagn 1999; 19: 142-145.

28 Snijders R: Fetal loss in Down syndrome pregnancies. Prenat Diagn 1999; 19: 1180

29 Spencer $\mathrm{K}$ : What is the true fetal loss rate in pregnancies affected by trisomy 21 and how does this influence whether first trimester detection rates are superior to those in the second trimester? Prenat Diagn 2001; 21: 788-789.

30 Wald N Rodeck C, Hackshaw AK, Walters J, Chitty L, Mackinson AM, Bestwick JP: Correction to SURUSS report. J Med Screen 2006; 13: 51-52.

31 Malone FD, Canick JA, Ball RH et al: First-trimester or second-trimester screening, or both, for Down's syndrome. N Engl J Med 2005; 353: 2001-2011.

32 Leung WC, Lam YH, Wong Y, Lau ET, Tang MH: The effect of fast reporting by amnioPCR on anxiety levels in women with positive biochemical screening for Down syndrome - a randomized controlled trial. Prenat Diagn 2002; 22: 256-259.

33 Chitty LS, Kagan KO, Molina FS, Waters JJ, Nicolaides KH: Fetal nuchal translucency scan and early prenatal diagnosis of chromosomal abnormalities by rapid aneuploidy screening: observational study. BMJ 2006; 332: 452-455.

34 Buckley F, Buckley SJ: Costs of prenatal genetic screening. Lancet 2008; 372 : 1789-1791.

35 Wald N, Rodeck C, Rudnicka A, Hackshaw A: Nuchal translucency and gestational age. Prenat Diagn 2004; 24: 150-151.

36 Nicolaides $\mathrm{KH}$, Spencer K, Avgidou K, Faiola S, Falcon 0: Multicenter study of firsttrimester screening for trisomy 21 in 75821 pregnancies: results and estimation of the potential impact of individual risk-orientated twostage first-trimester screening. Ultrasound Obstet Gynecol 2005; 25: 221-226.

37 Sundberg K, Bang J, Smidt-Jensen S et al: Randomised study of risk of fetal loss related to early amniocentesis versus chorionic villus sampling. Lancet 1997; 350: 697-703.

38 Jacobs PA, Browne $\mathrm{C}$, Gregson $\mathrm{N}$, Joyce $\mathrm{C}$, White $\mathrm{H}$ : Estimates of the frequency of chromosome abnormalities detectable in unselected newborns using moderate levels of banding. J Med Genet 1992; 29: 103-108.

39 Knight SJ, Regan R, Nicod A et al: Subtle chromosomal rearrangements in children with unexplained mental retardation. Lancet 1999; 354: 1676-1681.

40 Miller DT, Adam MP, Aradhya S et al: Consensus statement: chromosomal microarray is a first-tier clinical diagnostic test for individuals with developmental disabilities or congenital anomalies. Am J Hum Genet 2010; 86: 749-764.

41 Seror V: Fitting observed and theoretical choices - women's choices about prenatal diagnosis of Down syndrome. Health Econ 2008; 17: 557-577.

42 Wald NJ, Watt HC, Hackshaw AK: Integrated screening for Down's syndrome on the basis of tests performed during the first and second trimesters. N Eng/ J Med 1999; 341: 461-467.

43 Faucher E, Bujold E, Brassard N, Himaya E, Gekas J: FISH analysis for fetal karyotype : what are the factors associated with its failure? Prenat Diagn 2008; 28: S27.

44 Bestwick JP, Wald N, Rudnicka AR: Cost of integrated, sequential and contingent prenatal screening for Down's syndrome. BMJ 2009; e-pub ahead of print 2 April 2009.

45 Midtrimester amniocentesis for prenatal diagnosis: Safety and accuracy. JAMA 1976; 236: $1471-1476$

46 Alfirevic Z, Sundberg K, Brigham S: Amniocentesis and chorionic villus sampling for prenatal diagnosis. Cochrane Database Syst Rev 2003; (3): CD003252.

47 Mujezinovic F, Alfirevic Z: Procedure-related complications of amniocentesis and chorionic villous sampling: a systematic review. Obstet Gynecol 2007; 110: 687-694.

48 Tabor A, Alfirevic Z: Update on procedure-related risks for prenatal diagnosis techniques. Fetal Diagn Ther 2009; 27: 1-7.

49 Tabor A, Vestergaard $\mathrm{CH}$, Lidegaard O: Fetal loss rate after chorionic villus sampling and amniocentesis: an 11-year national registry study. Ultrasound Obstet Gynecol 2009; 34: 19-24.

This work is licensed under the Creative Commons Attribution-NonCommercial-No Derivative Works 3.0 Unported Licence. To view a copy of this licence, visit http:// creativecommons.org/licenses/by-nc-nd/3.0/ 